\title{
Preliminary results of Galileo direct imaging of S-L 9 impacts
}

\author{
Clark R. Chapman', William J. Merline', Kenneth Klaasen², Torrence V. \\ Johnson $^{2}$, Catherine Heffernan ${ }^{2}$, Michael J. S. Belton ${ }^{3}$, Andrew P. Ingersoll ${ }^{4}$, \\ and the Galileo Imaging Team
}

\begin{abstract}
Direct Galileo imaging data were obtained of the Jupiter impact sites for Comet Shoemaker-Levy 9 fragments $K, N$, and $W$ during their early, high-energy phases. Initial $\sim 5$ s-long flashes for all 3 impacts result from radiant bolides; analogous, abrupt onsets of luminosity observed by the Galileo photopolarimeter for other impacts must also be the bolide phase. The 3 bolides were $\operatorname{dim}$ at 0.56 or $0.89 \mu \mathrm{m}$ (few percent of total Jupiter) and had similar amplitudes, despite huge latestage differences observed from Earth. Subsequent, continuous luminosity lasting $\sim 40$ s for $\mathrm{K}$ and $\sim 10$ s for $N$ is optical radiation as the initial bolide train erupts into a "fireball". The K light curve may show (a) two impacts 10 s apart or (b) delayed evolution of the fireball.
\end{abstract}

\section{Introduction}

The Solid State Imaging (SSI) camera on Galileo was the only instrument to image the actual impact sites of Shoemaker-Levy 9 (SL9) fragments as they struck. This is our first report on data and interpretations. Data for impacts $\mathrm{K}$ and $\mathrm{N}$ became available in October; final tape recorded W images were returned in January 1995. SSI data strongly constrain the early, highest energy phases of the impacts, which were hidden from Earth behind Jupiter's limb.

We used two observing modes to cover the diverse phenomena predicted for these unprecedented comet impacts. Both took advantage of a new capability (onchip mosaicking) developed by Galileo engineers to permit multiple-exposure imaging. The SSI can shutter as often as every $21 / 3$ seconds; for $W$, we recorded $7 \times 8$ arrays of time-lapse images on each frame. To see more rapid time variations in impact light curves-for example, impacts of fragment-cluster components (Weidenschilling, 1994; Asphaug \& Benz, 1994)-we designed a scan mode, used for the $K$ and $N$ events; this mode also efficiently used Galileo's limited downlink capacity.

SL9 impacts observed by SSI were not studied by the other scan platform instruments (Ultraviolet Spectrometer [UVS], Photopolarimeter Radiometer [PPR], and Near Infrared Mapping Spectrometer [NIMS]), which focussed on a complementary set of impacts, because the on-chip mosaic mode is incompatible with non-SSI ob-

\footnotetext{
${ }^{1}$ Planetary Science Inst., Tucson, AZ

${ }^{2}$ Jet Propulsion Lab., Caltech, Pasadena, CA

${ }^{3}$ National Optical Astronomy Observatories, Tucson, AZ

${ }^{4}$ Division of Geological Sciences, Caltech, Pasadena, CA
}

Copyright 1995 by the American Geophysical Union.

Paper number 95GL01030

0094-8534/95/95GL-01030\$03.00 serving modes. Of the four impacts for which SSI took data, downlink resources were devoted primarily to $K$ and $W$ plus return of a single frame for the weak $N$ impact. We decided not to return data from $V$.

Galileo was 1.60 AU from Jupiter, at an angle of $4012^{\circ}$ to the Earth-Jupiter line and $51^{\circ}$ to the Sun-Jupiter line. Gibbous Jupiter was 59 pixels across $(2430 \mathrm{~km} /$ pixel resolution), with the impact sites in direct view on the southern, pre-dawn night side. After discussing our $\mathbf{K}$, $\mathrm{N}$, and $\mathrm{W}$ data separately, we compare and synthesize our results with other Galileo data and Hubble Space Telescope (HST) images of early-stage phenomena for these and other impacts.

\section{Impact of Fragment $\mathrm{K}$}

For K, the shutter was opened for its maximum duration, 25.6s, while the scan platform was moved to drift Jupiter across the CCD frame. After a 5s reposition slew, another $25.6 \mathrm{~s}$ swath was obtained and so on for 5 or 6 swaths, totalling 21/2-3 min. After a gap of 35-65s for read-out, a new multiple-swath frame began. Scanning yields a time-resolution of $0.072 \mathrm{~s}$ per CCD line. Scans were oriented diagonally so that Jupiter swaths would not overlap trails of impact phenomena adjacent to Jupiter's morning terminator.

$\mathrm{K}$ and $\mathrm{N}$ were observed through a narrow (FWHM $\sim 0.017 \mu \mathrm{m}$ ) methane-band filter centered at $0.89 \mu \mathrm{m}$ to permit a reasonable scan rate without saturating Jupiter. The methane absorption also dims Jupiter -5-10 times compared with the adjacent continuum, enhancing the contrast of high-altitude impact phenomena.

This observing mode yields several artifacts. Because of multiple exposures, trails of Galilean satellites from nearby scans can overlap Jupiter or impact sites. Also, spacecraft spin induces scan platform motion, resulting in obvious left-right wiggles and variations in scan rate (perpendicular to the wiggles) that affect photometry.

The scan mode was programmed, in the command sequence readied for uplink in June 1994, to execute for $2 \mathrm{hr}$ bracketing the expected $\mathrm{K}$ impact time. Refined predictions of 7 July 1994 enabled us to select a portion, 9:54:23-10:54:28 on 19 July 1994, to be recorded. (Throughout this paper, times are the equivalent UTC for a groundbased observer.) Based on returned jail-bar samples (groups of contiguous data at widely-spaced, regular intervals returned to locate data on the tape) covering 10:16-10:37, plus Earthbased data, we decided to return 3 frames (10:19:39-10:29:41) plus additional less-dense jail-bars for 2 frames (10:30:16-10:36:46).

Already in early August, the jail-bars for 10:23:1210:25:39 showed the $\mathrm{K}$ impact prominently, despite coarse time resolution. Detection was announced on the 
SL9 Exploder on 12 Aug. 1994 and posted on the World Wide Web (http://www.noao.edu/galileo/s19/s19.html; hereafter WWW). Remaining $\mathrm{K}$ data were returned during October 1994. They have periodic gaps to conserve downlink resources; although more data were actually transmitted to Earth, here we analyze groups of 2-4 lines on 8-line centers. Groups look like narrow bars, which repeat every $0.58 \mathrm{~s}$; within a bar, time samples are separated by $0.072 \mathrm{~s}$.

Fig. 1 depicts the frame showing the $\mathrm{K}$ event (see also WWW). A prominent luminous event begins near the top of swath 3 from the left and continues through most of swath $4 . \quad K$ begins at 10:24:13 and lasts for $-52 \mathrm{~s}$ (timing within each bar is known to $\pm 2 \mathrm{~s}$ ). The trail width is consistent with an unresolved point source.

Fig. 2 shows a light curve for $K$. The precision is high, except where scan platform wobble briefly brings the $\mathrm{K}$ trail close to Jupiter; open symbols indicate less reliable background-subtraction near Jupiter. We corrected for uneven scan platform motion based on photometry of trails of Io and Ganymede (which cannot vary during 30s). The trails show a pattern of nearly identical right-left oscillations, defining a $19.6 \mathrm{~s}$ period, which is the spacecraft spin period. Satellite trails from the three most complete frames were co-added in phase, yielding correction factors of $\pm 20 \%$. Unfortunately, the combined satellite signal is too weak to define corrections to better than $\pm 5 \%$ for each $1 / 3 \mathrm{~s}$ interval. Nevertheless, smaller spikes in the raw data are clearly due to the nonuniform scanning rates. We have smoothed the correction factors with a spline fit, which, in turn, was applied to $\mathrm{K}$ data at the appropriate phase.

The $\mathrm{K}$ light curve shows a clearly real initial "flash" with FWHM of $-5 \mathrm{~s}$, which begins with an abrupt rise, most of which takes only $2 \mathrm{~s}$. Despite its modest signature in the raw data, the second, abrupt $30 \%$ rise $15 \mathrm{~s}$ into the event may be real. It is followed by a gradual decline. The light curve has a "spiky" aspect, which-if real-might indicate impacts of multiple fragments. But we believe small variations are due to slight non-repeatability of the scan platform motion plus noise in the correction factors due to the low satellite signal level. Some effects may be due to background subtraction.

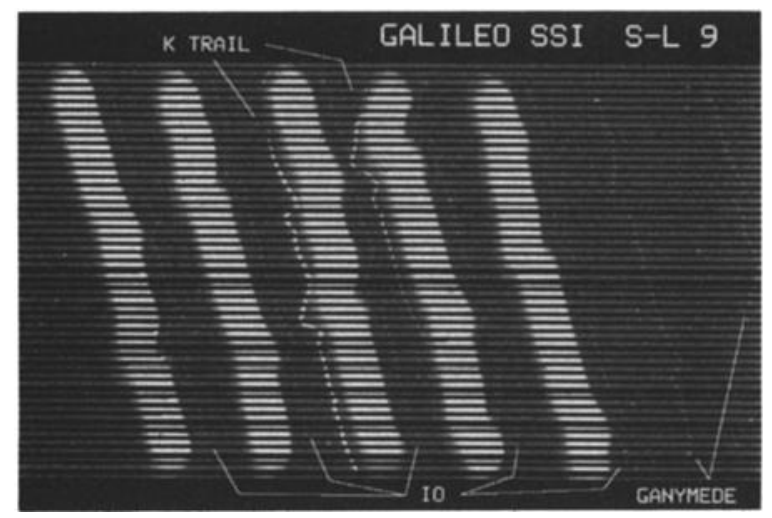

Figure 1. SSI frame showing $K$ impact. The five swaths are Jupiter, beginning at the upper left, progressing to the lower right. Trail for the $\mathrm{K}$ impact is noted, as are trails for Io and Ganymede. See text for details.

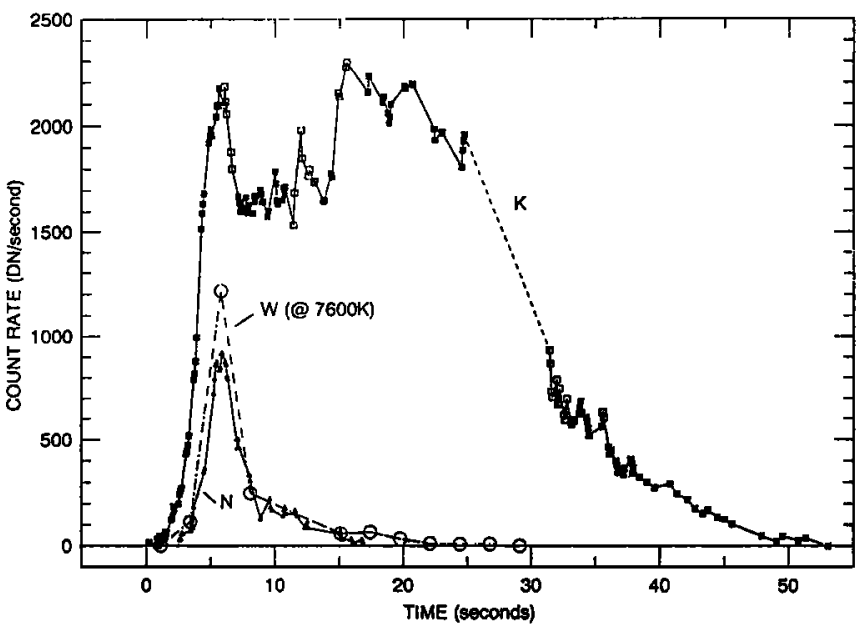

Figure 2. SSI light curves for the $K, N$, and $W$ impacts. $W$ data are scaled to the others assuming a $7600 \mathrm{~K}$ black body (see text). Open circles for $\mathrm{K}$ have larger uncertainties in background subtraction than solid points. Dashed line is the gap between two swaths. $\mathrm{K}$ data (but not $N$ ) have been corrected for irregular scan platform motion. Note the similarity in shape of the initial peaks, which we interpret to be bolide flashes. $1 \mathrm{DN} / \mathrm{s}$ is a flux density of $0.73 \times 10^{-18} \mathrm{~W} \mathrm{~cm}^{-2} \mathrm{~nm}^{-1}$ at Galileo's distance from Jupiter, $1.60 \mathrm{AU}$.

The gross shape and duration of the $\mathrm{K}$ light curve resembles that observed by the Galileo PPR, at a similar wavelength, for impacts G, H, L, and Q1 (Martin et al., 1994). PPR data have lower $S / N$ than SSI, which shortens apparent PPR event durations. Widths at half height show that K's duration (27s) equals the longest observed by PPR (L) and exceeds $G, H$, and Q1. The peak brightness of $\mathrm{K}$ was $~ 19 \%$ of total Jupiter in our methane band filter (it would be 5-10 times less in the neighboring continuum).

We find no phenomena in the 3 frames following the one showing the impact, despite hints at $1 \mathrm{DN}$ above background. Prominent infrared phenomena were seen from Earth during our last two frames. D. Crisp et al. (SL9 Exploder, July 1994) reported that the K plume was 400 times brighter than Jupiter's south polar hood, in a $2.34 \mu \mathrm{m}$ methane filter, at a time near the end of our penultimate frame. Of course, while the plume was rotating into view from Earth, it moved closer to Jupiter's terminator as seen from Galileo, hence closer to our trailed Jupiter swaths. We have not yet quantified our detection threshold, but our data show that the $K$ plume probably could not have exceeded $1 / 4$ the brightness of Io through 10:33, which may constrain plume dimensions, optical depth, and/or albedo.

The $\mathrm{K}$ event observed by SSI must be the high-energy, luminous phase of the impact, though at least two independent Earthbased observations show events $3 \mathrm{~min}$ earlier. "The first indication of a plume was detected at UT 10:21:22" at $2.34 \mu \mathrm{m}$ according to an early Exploder report (P. McGregor \& M. Allen). One SSI image covers a 3-min period centered on 10:21, with one swath during 10:21:10-10:21:36, yet we see no luminous event brighter than $10 \%$ of Io in this swath or in the entire frame; it is unlikely that one would have occurred wholly 
within a 5-s-long gap between swaths. Our upper limit is not inconsistent with the McGregor \& Allen report, given the extraordinary sensitivity of their $2 \mu \mathrm{m}$ methaneband technique; they may have seen a precursor coma impact. Also, Waite et al. (1994) report a sudden enhancement in X-ray flux, from a place in Jupiter's northern hemisphere that is roughly the magnetic field conjugate of the impact site, starting at $10: 21 \pm 1 \mathrm{~min}$. It is not known how the $\mathrm{X}$-ray flash is related to the $\mathrm{K}$ impact.

\section{Impact of Fragment $\mathbf{N}$}

The $\mathrm{N}$ impact was observed in the same scan mode and filter as K. Only one frame was returned to Earth; 5 swaths cover 10:28:13-10:30:40 UTC on 20 July 1994. It was posted on WWW in November. Generally only 4 of every 12 lines were transmitted to Earth, with gaps between groups of lines no longer than $0.6 \mathrm{~s}$ duration. A luminous event is visible from 10:29:17-10:29:32.

There is slight confusion from proximate multipleexposure trails of Io and Europa, though they are much fainter than the main event (except near its end) and can be separated by the well-defined pattern of scan-platform motion. Data quality during the event is little affected by the trails, but detectability of marginal events at other times is somewhat compromised. We see no such events. The $\mathrm{N}$ light curve in Fig. 2 has not yet been corrected for scan platform motion, so $\pm 20 \%$ variations may exist. Still, its form is clear: a sharp flash with a rise time of $\sim 3 \mathrm{~s}$ and a duration of $6 \mathrm{~s}$, followed by a faint $9 \mathrm{~s}$ trailing off. $\mathrm{N}$ peaks at $\sim 40 \%$ of $\mathrm{K}(\sim 8 \%$ of total Jupiter, in the methane filter), but the faint glow 5-10s later is only $5 \%-10 \%$ of $\mathrm{K}$ 's brightness at an equivalent time.

From Earth, $\mathrm{N}$ seemed minimal $(2 \mu \mathrm{m}$ brightness $\sim 600$ times less than K; D. Crisp et al., SL9 Exploder, July 1994) and it left only a small spot on Jupiter as imaged by HST. No other phenomena were reported near the time of the SSI event, which is well within the severalminute probable impact interval estimated from astrometry of $\mathrm{N}$ in the context of other SL9 phenomenology (10:31, P. Chodas and D. Yeomans, priv. comm. 2 Aug. 1994) and from backward extrapolation from the longitude of the HST spot (10:30, Hammel et al., 1994).

\section{Impact of Fragment $W$}

Here we chiefly discuss a few test images returned in early August, which luckily showed the impact. Unlike the scan mode used for $\mathrm{K}$ and $\mathrm{N}, \mathrm{W}$ was directly imaged every $21 / 3$ s (time-lapse). With on-chip mosaicking, each frame has 7 rows of 8 images each. There is usually a $7 \mathrm{~s}$ gap between rows and $86 \mathrm{~s}$ between frames. 1800 images were shuttered for $W$, but just a fraction of the 30 frames were recorded, and only some of that data (swaths across $\sim 19$ rows of 8 images each) was returned.

Each image is an $81 / \mathrm{s} \mathrm{ms}$ exposure through the green filter $(0.56 \mu \mathrm{m})$. Four images (posted on WWW) were widely published: the last four in a sequence of 8 that began at 8:06:02. Images through 8:06:12 show no event, but a bright source on Jupiter's dark side is in the final three images. A preliminary position (lat. $-43^{\circ}$, long. $280^{\circ}$ Sys. III) is near that $\left(-44^{\circ}, 280^{\circ}\right)$ predicted by $P$. Chodas and D. Yeomans (priv. comm.). (The SSI im- pact time was $\sim 5$ min later than predicted; the resulting $4^{\circ}$ longitude discrepancy is within measurement and prediction errors, however.)

W brightened and then faded over $7 \mathrm{~s}$. To plot $\mathrm{W}$ in Fig. 2, we take into account the different observing mode and assume a color dependence of the flux; here we adopt a $7600 \mathrm{~K}$ black body, as measured by UVS/PPR for $G$ during its first few seconds (cf. Martin et al., 1994; for $20,000 \mathrm{~K}$, W would be about half as bright relative to $\mathrm{K}$ and $\mathrm{N}$ ). Correcting for saturation of the central pixel, we find that $\mathrm{W}$ peaked at visual magnitude +0.55 as seen from Galileo, compared with -4.0 for Jupiter itself. Thus $\mathrm{W}$ reached only $\sim 1.5 \%$ of the brightness of Jupiter in green, unexpectedly weak for an impact that generated an impressive plume imaged by HST (Hammel et al., 1994). After the $7 \mathrm{~s}$ gap following this row, $W$ fades away in the first 6 images of the next row, returned in January 1995. Despite better sensitivity to faint phenomena in this time-lapse mode compared with the scan mode, no development of the $\mathrm{W}$ plume is seen in preliminary inspection of the January data.

HST imaged $W-$ also in green light-at the identical time (to within the 1s timing uncertainties) of the SSI image at peak brightness (Hammel et al., 1994). (The coincidence is amazing since HST images were typically 3 min apart.) HST shows a source within Jupiter's shadow, which must either be luminous (e.g. the entry bolide above the $\sim 160 \mathrm{~km}$ level of the limb projection) or a reflection of a lower-altitude luminous event from some material, perhaps comet dust. Given that our W event is the bolide flash (see below), the HST image must show either the high-altitude phase of the bolide or its indirect reflection.

\section{Interpretation and Conclusions}

Zahnle and Mac Low (1994) predicted an initial bolide flash, followed after -10 s of darkness by a fireball erupting from below Jupiter's clouds. Alternatively, Boslough et al. (1994) calculated that the high temperature trail in Jupiter's stratosphere left by the bolide immediately explodes, forming the visible top of the ascending fireball; one can infer from their calculations that there should be no intervening gap between the bolide flash and the developing fireball luminosity. Still others (T. Martin, G. Orton, priv. comm., 1995) suggest that the bolide phase might have been invisibly dim, so that all luminous phenomena during the first minutes are from the rising fireball. (The prominent luminosity observed at $2 \mu \mathrm{m}$ from Earth $5-20 \mathrm{~min}$ later was due to re-impacting plume debris.)

SSI data immediately settle this debate. In Fig. 2, each of the three superimposed light curves shows an initial peak (or "flash") $\sim 5$ s long. All show an abrupt, dramatic rise (most of it over $2 s$ ) to that initial peak, followed by a sudden decay before the onset of a more gentle decay. This initial flash must be the entry bolide, for several reasons. Our pre-impact coverage of $K$ and $\mathrm{N}$ is good enough to preclude the likelihood of any precursor bolide, unless it was extremely faint. The initial flash is not credible as a fireball bursting up through Jupiter's clouds, because there is no reason to expect it to then suddenly decay (followed, in the case of 
$\mathrm{K}$, by a subsequent brightening). Based on NIMS spectra, Carlson et al. (1994) show that the portion of the G light curve that is analogous to the long-lived component of our $\mathrm{K}$ light curve is, in fact, a growing, rising, and cooling fireball.

SSI data for the minor $\mathrm{N}$ impact bolster our view that the luminous phase of major SL9 impacts is the merger of two components-bolide and fireball-but without an intervening gap: N's initial flash (bolide) dominates, and the longer-lasting component (a weak fireball?) is very subdued. The initial UVS/PPR observation of a high temperature $(T \sim 7600 \mathrm{~K})$ source for $G$ is in the part of the light curve that we ascribe to the bolide.

The peak flux densities of the bolides, at the wavelengths observed (based on recent SSI stellar calibrations), are $2.0,1.6$, and $0.67 \times 10^{-15} \mathrm{~W} \mathrm{~cm}^{-2} \mathrm{~nm}^{-1}$ for $\mathrm{W}, \mathrm{K}$, and $\mathrm{N}$, respectively (or $0.66,1.4$, and $0.57 \times 10^{-15} \mathrm{~W} \mathrm{~cm}^{-2} \mathrm{~nm}^{-1}$ at the PPR wavelength of $0.945 \mu \mathrm{m}$, scaled assuming the UVS/PPR black body color temperature of $7600 \mathrm{~K}$ ). This places $\mathrm{K}$ between $\mathrm{Q} 1$ and $\mathrm{H}$ in energy, and $\mathrm{W}$ and $\mathrm{N}$ at about half Q1. Two caveats are (a) temperatures of different bolides may not be the same and (b) if bolide flashes were dominated by line emissions (e.g. $\mathrm{H} \alpha$ ), black body assumptions would not apply.

An unexpected SSI result is the apparent similarity in brightness of the bolide phases of our three impacts that, by other criteria (e.g. Earthbased infrared plume fluxes and remnant spot dimensions), ranged from among the strongest $(K)$, to middling $(W)$, to very weak $(N)$. Indeed, the 7 impacts observed by PPR or SSI vary by only a factor of 6 in peak energy during the bolide phase. Perhaps similar bolide luminosities are related to the unexpected similarity in plume heights observed for 4 events by HST (Hammel et al., 1994). If bolide radiance is a strong function of impactor dimension (rather than mass), then $\mathrm{K}$ could have been massive while $\mathrm{N}$ was underdense (perhaps a swarm). Alternatively, temperatures or opacities of the bolide phenomenon may be scale dependent, so that very different sized objects yield similar bolides (i.e. the physics embodied in the term "luminous efficiency" may be complicated).

The luminous events for $K$ and $W$ seem faint given their subsequent dramatic plumes and extensive debris aprons. Integrating over a $7600 \mathrm{~K}$ black-body Planck function for the duration of the bolide flash, we find total luminous energies only $-10^{23}$ ergs for the SSI events. If impactor kinetic energies were $10^{27} \cdot 10^{28} \mathrm{ergs}$, then bolide luminous efficiencies (at least at continuum wavelengths) were of order $0.01 \%$ or less, much lower than adopted by Zahnle and Mac Low (1994) for the bolide phase (they assumed higher temperature bolides). Of course, there was additional luminosity (especially for $\mathrm{K}$, only a little for $\mathrm{N}$ ) during the fireball phase, but that was while the temperatures were rapidly falling and the peak radiation was moving into the infrared. Probably, luminous energies were low in part because most impactor kinetic energy was deposited deep below Jupiter's cloud deck. The fraction of energy deposited in the stratosphere, however small, was evidently sufficient to produce the dramatic visible fireballs and plumes.

The $\mathrm{K}$ light curve differs somewhat in shape from PPR data for $G, H$, and $L$. Rather than decaying monotonically from the initial bolide flash, $\mathrm{K}$ brightens again and peaks some $15 \mathrm{~s}$ later. If our tentatively-identified second abrupt rise is real, then the $\mathrm{K}$ light curve could be the superposition of two separate impacts 10s apart (as if the PPR curve for Q1 were added to $\mathrm{H} 10$ s after $\mathrm{H}$ began). This would also help explain the longer duration of $K$ compared with the PPR events of comparable energy. Alternately, the second rise could be due to the emergence of a deep component of the fireball, as in case W1 of Zahnle and Mac Low (1994). Additional K data decoded in February 1995 (included in Fig. 1 but not yet analyzed) could help us choose between the alternatives.

Acknowledgments. We thank all SL9 observers for their accurate, timely reports, which were essential to the success of the Galileo experiments. We particularly thank P. Chodas and D. Yeomans of JPL for their tireless efforts to provide the latest, best ephemerides and timing information. We thank H. Hammel, G. Orton, M. Boslough, K. Zahnle, and M.-M. Mac Low for discussions. W. Cunningham (JPL) led the Galileo engineers who developed, tested, and loaded the on-chip mosaicking flight software ahead of schedule, enabling our experiment. Finally, we appreciate all the women and men of the Galileo Project, who took on the challenge of observing SL9 on short notice, while preparing for Galileo's prime Jupiter mission under trying circumstances. They made it happen. This is Contribution \#328 of the Planetary Science Institute, a division of San Juan Capistrano Research Institute. The Galileo Project is managed by the Jet Propulsion Laboratory, California Institute of Technology, under a contract with NASA.

\section{References}

Asphaug, E. \& W. Benz, Density of comet ShoemakerLevy 9 deduced by modelling breakup of the parent 'rubble pile', Nature, 370, 120-123, 1994.

Boslough, M., D.A. Crawford, A.C. Robinson, \& T.G. Trucano, Watching for fireballs on Jupiter, EOS, 75, 305, $307 \& 310,1994$.

Carlson, R.W. et al., Galileo infrared observations of the Shoemaker-Levy $9 \mathrm{G}$ impact fireball: a preliminary report, Geophys. Res. Lett., this issue, 1995.

Hammel, H.B. et al., HST imaging of atmospheric phenomena created by the impact of comet Shoemaker-Levy 9, Science 267, 1288-1296, 1994.

Martin, T.Z. et al., Galileo PPR observations of ShoemakerLevy 9, B.A.A.S., 26, 1566, 1994.

Waite, J.H. Jr. et al., $X$ ray emissions produced as a result of the impact of comet Shoemaker-Levy 9 with Jupiter, $B$ A.A.S., in press, 1994.

Weidenschilling, S. J., Origin of cometary nuclei as 'rubble piles', Nature, 368, 721-723, 1994.

Zahnle, K. and Mac Low, M.-M., The collision of Jupiter and comet Shoemaker-Levy 9, Icarus, 108, 1-17, 1994.

Clark R. Chapman and William J. Merline, Planetary Science Inst., 620 N. 6th Ave., Tucson, AZ 85705. (e-mail: cchapman@psi.edu, merline@psi.edu)

Kenneth Klaasen (MS 306-431), Torrence V. Johnson (MS 183-501), and Catherine Heffernan (MS 264-765), Jet Propulsion Lab., 4800 Oak Grove Dr., Pasadena, CA 91109. (e-mail: Kenneth.P.Klaasen@jpl.nasa.gov, tjohnson@jpltvj. jpl.nasa.gov, and Catherine.M.Heffernan@jpl.nasa.gov)

Michael J. S. Belton, Natl. Optical Astronomy Obs., P.O. Box 26732, Tucson, AZ 85726. (e-mail: belton@noao.edu)

Andrew T. Ingersoll, Div. of Geol. \& Plan. Sci., MS 17025, Calif. Inst. of Technology, Pasadena, CA 91125. (e-mail: api@satur1.gps.caltech.edu)

(Received December 20, 1994; accepted February 20, 1995.) 